\title{
Coefficient Problem for Certain Classes of Analytic Functions using Hankel Determinant
}

\section{B. Srutha Keerthi, S. Prema}

Abstract: In this paper, we introduce some classes of analytic-univalent functions and for any real $\mu$, determine the sharp upper bounds of the functional $\left|a_{2} a_{4}-\mu a_{3}^{2}\right|$ for the functions of the form $f(z)=z+\sum^{\infty} a_{k} z^{k}$

$k=2$

belonging to such classes in the unit disc $E=\{z:|z|<1\}$.

Keywords: Analytic functions, functions with positive real part, starlike functions with respect to symmetric points, convex functions with respect to symmetric points, Hankel determinant.

Mathematics Subject Classification: $30 \mathrm{C45}$.

\section{INTRODUCTION}

Let $A$ be the class of analytic functions of the form

$$
\underset{k=2}{f(z)=z+a_{k} z^{k}}
$$

in the unit disk $\Delta=\{z:|z|<1\}$.

Let $S$ be the class of functions $f(z) \in A$ and univalent in $\Delta$. In the present paper, we consider the following subclasses of $A$.

\section{Definition 1.1.}

Let

$S_{S(\lambda)}^{*}=\left\{f \in A, R e\left[\frac{2\left[\lambda z^{2} f^{\prime \prime}(z)+z f^{\prime}(z)\right]}{\lambda z\left[f^{\prime}(z)+f^{\prime}(-z)\right]+(1-\lambda)[f(z)-f(-z)]}\right]>0,0 \leq \lambda \leq 1\right\}$

the class of starlike functions with respect to symmetric points.

$K_{S(\lambda)}=\left\{f \in A, \operatorname{Re}\left[\frac{2\left[\lambda z^{2} f^{\prime \prime}(z)+z f^{\prime}(z)\right]^{\prime}}{\left[\lambda z\left[f^{\prime}(z)+f^{\prime}(-z)\right]+(1-\lambda)[f(z)-f(-z))\right]^{\prime}}\right]>0,0 \leq \lambda \leq 1\right\}$

the class of convex functions with respect to symmetric points.

$C_{S(\lambda)}=\left\{f \in A, R e\left[\frac{2\left[\lambda z^{2} f^{\prime \prime}(z)+z f^{\prime}(z)\right]}{\lambda z\left[g^{\prime}(z)+g^{\prime}(-z)\right]+(1-\lambda)[g(z)-g(-z)]}\right]>0, g \in S_{S(\lambda)}^{*}, z \in \Delta\right\}$

the class of close-to-convex functions with respect to

symmetric points.

Revised Manuscript Received on July 08, 2019.

B. Srutha Keerthi ${ }^{1}$ School of Advanced Sciences Mathematics Division, VIT University, Chennai Campus,Vandalur, Kelambakkam, Chennai - 600127 sruthilaya06@yahoo.co.in

S.Prema, ${ }^{2}$ Department of Mathematics,SRM Institute of Science and Technology,Chennai - 600089 cs.prem@ yahoo.co.in
The classes $K_{S}$ and $C_{S}$ were introduced by Das and Singh

[2].

$C_{S 1(\lambda)}=\left\{f \in A, R e\left[\frac{2\left[\lambda z^{2} f^{\prime \prime}(z)+z f^{\prime}(z)\right]}{\lambda z\left[h^{\prime}(z)+h^{\prime}(-z)\right]+(1-\lambda)[h(z)-h(-z)]}\right]>0, h \in K_{S(\lambda)}, z \in \Delta\right\}$

$C_{S 1(\lambda)}^{\prime}=\left\{f \in A, R e\left[\frac{2\left[\lambda z^{2} f^{\prime \prime}(z)+z f^{\prime}(z)\right]^{\prime}}{\left[\lambda z\left[h^{\prime}(z)+h^{\prime}(-z)\right]+(1-\lambda)[h(z)-h(-z)]\right]^{\prime}}\right]>0, h \in K_{S(\lambda)}, z \in \Delta\right\}$

\section{Definition 1.2.}

Let

$S_{S(\gamma, \lambda)}=\left\{f \in A: R e\left[\begin{array}{r}\frac{2\left[\gamma \lambda z^{3} f^{\prime \prime \prime}(z)+(2 \gamma \lambda+\gamma-\lambda) z^{2} f^{\prime \prime}(z)+z f^{\prime}(z)\right]}{\gamma \lambda z^{2}\left[f^{\prime \prime}(z)-f^{\prime \prime}(-z)\right]+(\gamma-\lambda) z\left[f^{\prime}(z)+f^{\prime}(-z)\right]} \\ +(1-\gamma+\lambda)[f(z)-f(-z)] \quad 0 \leq \lambda \leq \gamma \leq 1\end{array}\right]>0\right.$

the class of starlike functions with respect to symmetric points

$K_{S(\gamma, \lambda)}=\left\{f \in A: R e\left[\begin{array}{c}(1.1) \\ {\left[\begin{array}{c}2\left[\gamma \lambda z^{3} f^{\prime \prime \prime}(z)+(2 \gamma \lambda+\gamma-\lambda) z^{2} f^{\prime \prime}(z)+z f^{\prime}(z)\right]^{\prime} \\ {\left[\gamma z^{2}\left[f^{\prime \prime}(z)-f^{\prime \prime}(-z)\right]+(\gamma-\lambda) z\left[f^{\prime}(z)+f^{\prime}(-z)\right]\right.}\end{array}\right]>0} \\ +(1-\gamma+\lambda)[f(z)-f(-z)]]^{\prime} \quad \\ \quad 0 \leq \lambda \leq \gamma \leq 1\end{array}\right\}\right.$

the class of convex functions with respect to symmetric points.

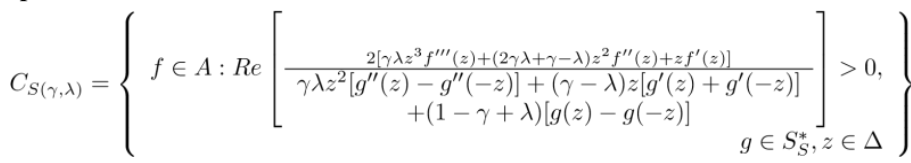

the class of close-to-convex functions with respect to symmetric points.

$C_{S 2(\gamma, \lambda)}=\left\{f \in A: \operatorname{Re}\left[\begin{array}{c}\frac{2\left[\gamma \lambda z^{3} f^{\prime \prime \prime}(z)+(2 \gamma \lambda+\gamma-\lambda) z^{2} f^{\prime \prime}(z)+z f^{\prime}(z)\right]}{\gamma \lambda z^{2}\left[h^{\prime \prime}(z)-h^{\prime \prime}(-z)\right]+(\gamma-\lambda) z\left[h^{\prime}(z)+h^{\prime}(-z)\right]} \\ +(1-\gamma+\lambda)[h(z)-h(-z)] \quad\end{array}\right]=0, \quad h \in K_{S}, z \in \Delta\right\}$

$C_{S 2(\gamma, \lambda)}^{\prime}=\left\{f \in A: \operatorname{Re}\left[\begin{array}{r}\frac{2\left[\gamma \lambda z^{3} f^{\prime \prime \prime}(z)+(2 \gamma \lambda+\gamma-\lambda) z^{2} f^{\prime \prime}(z)+z f^{\prime}(z)\right]^{\prime}}{\left[\gamma \lambda z^{2}\left[h^{\prime \prime}(z)-h^{\prime \prime}(-z)\right]+(\gamma-\lambda) z\left[h^{\prime}(z)+h^{\prime}(-z)\right]\right.} \\ +(1-\gamma+\lambda)[h(z)-h(-z)]]^{\prime}\end{array}\right]>0, \quad h \in K_{S}, z \in \Delta\right\}$

In 1976, Noonan and Thomas [12] stated the $q^{\text {th }}$ Hankel determinant for

$q \geq 1$ and $n \geq 1$ as 
$H q(n)=\left|\begin{array}{cccc}a_{n} & a_{n+1} & \ldots & a_{n+q+1} \\ a_{n+1} & \ldots & & \ldots \\ a_{n+q+1} & \ldots & & a_{n+2 q-2}\end{array}\right|$

This determinant has also been considered by several authors, for example, Noor [13] determined the rate of growth at $H q(n)$ as $n \rightarrow \infty$ for functions given by Equation (1.1) with bounded boundary. Ehrenborg [3] studied the Hankel determinant of exponential polynomials and in [6], the Hankel transform of an integer sequence is defined and some of its properties discussed by Layman.Also Hankel determinant was studied by various authors including Hayman [5] and Pommerenke [14] and recently by Choc and Janteng [1], Mehrok and Singh [9] and Janteng et al. [10, 11].

Easily, one can observe that the Fekete and Szeg"o functional is $H_{2}(1)$. Fekete and Szeg"o [4] then further generalized the estimate $\left|a_{3}-\mu a_{2}^{2}\right|$ where $\mu$ is real and $f \in S$. For our discussion in this paper, we consider the Hankel determinant in the case of $q=2$ and $n=2$,

$$
\left|\begin{array}{ll}
a_{2} & a_{3} \\
a_{3} & a_{4}
\end{array}\right|
$$

In this paper, we seek upper bound for the functional $\left|a_{2} a_{4}-\mu a_{3}^{2}\right|$ where $\mu$ is real, for the functions belonging to the above defined classes.

\section{Preliminary Results}

Let $P$ be the family of all functions $p$ analytic in $\Delta$ for which $\operatorname{Re}(P(z))>0$ and

$$
P(z)=1+p_{1} z+p_{2} z^{2}+\cdots \text { for } z \in \Delta .(2.1)
$$

\section{Lemma 2.1. [14]}

If $p \in P$, then $\left|p_{k}\right| \leq 2(k=1,2,3, \ldots)$

Lemma 2.2. $[7,8]$

If $p \in P$, then $2 p_{2}=p^{2}{ }_{1}+\left(4-p^{2}{ }_{1}\right) x$

$$
4 p_{3}=p_{1}^{3}+2 p_{1}\left(4-p^{2}{ }_{1}\right) x-p_{1}\left(4-p_{1}{ }^{2}\right) x^{2}+2\left(4-p^{2}{ }_{1}\right)\left(1-|x|^{2}\right) z,
$$
for some $x$ and $z$, satisfying $|x| \leq 1,|z| \leq 1$ and $p_{1} \in[0,2]$.

\section{Main Result}

\section{Theorem 3.1.}

If $f(z) \in C_{S(\lambda)}$ then

$\int \frac{\left[(1+2 \lambda)^{2}-(1+\lambda)(1+3 \lambda) \mu\right]^{2}}{\left[(1+2 \lambda)^{2}-2(1+\lambda)(1+3 \lambda) \mu\right]}-(1+\lambda)(1+3 \lambda) \mu$

$$
\left|a_{2} a_{4}-\mu a_{3}^{2}\right| \leq \begin{cases}(1+2 \lambda)^{2}-(1+\lambda)(1+3 \lambda) \mu & i^{0} \leq \mu \leq \frac{1}{2} f \\ (1+\lambda)(1+3 \lambda) \mu & \text { if, if } \mu \geq 1 . \\ \frac{(1+\lambda)(1+3 \lambda) \mu-\left.(1+2 \lambda)^{2}\right|^{2}}{\left[(1+\lambda)(1+3 \lambda) \mu-(1+2 \lambda)^{2}\right]}+\mu(1+\lambda)(1+3 \lambda) & \end{cases}
$$

Proof:

Since $f \in C_{S}$, by definition we have $\frac{2\left[\lambda z^{2} f^{\prime \prime}(z)+z f^{\prime}(z)\right]}{\lambda z\left[g^{\prime}(z)+g^{\prime}(-z)\right]+(1-\lambda)[g(z)-g(-z)]}=p(z$

where

$$
g(z)=z+{ }^{\mathrm{X}} b_{k} z^{k} \in S_{S^{*}(\lambda)}
$$$$
\infty
$$

Using (1.1), (2.1) and (3.1), (3.2) gives

$$
\begin{aligned}
1 & +2(1+\lambda) a_{2} z+(1+2 \lambda) 3 a_{3} z^{2}+(1+3 \lambda) 4 a_{4} z^{3} \\
& =\left(1+p_{1} z+p_{2} z^{2}+\cdots\right)\left[1+(1+2 \lambda) b_{3} z^{2}+\cdots\right]
\end{aligned}
$$

On equating co-efficients in (3.9), we get

$$
\begin{gathered}
2(1+\lambda) a_{2}=p_{1}, \\
3 a_{3}(1+2 \lambda)=(1+2 \lambda) b_{3}+p_{2}, \\
4 a_{4}(1+3 \lambda)=(1+2 \lambda) p_{1} b_{3}+p_{3}
\end{gathered}
$$

From (3.2), we can easily verify that

$$
b_{3}=\frac{p_{2}}{2(1+2 \lambda)}
$$

So (3.4) yields

$a_{2}=\frac{p_{1}}{2(1+\lambda)}, \quad a_{3}=\frac{p_{2}}{2(1+2 \lambda)}, \quad a_{4}=\frac{p_{3}}{4(1+3 \lambda)}+\frac{p_{2} p_{1}}{8(1+3 \lambda)}$

(3.5) From (3.5),

$$
\left|a_{2} a_{4}-\mu a_{3}^{2}\right|=\left|\frac{p_{1} p_{3}}{8(1+\lambda)(1+3 \lambda)}+\frac{p_{2} p_{1}^{2}}{16(1+\lambda)(1+3 \lambda)}-\mu_{1} \frac{p_{2}^{2}}{4(1+2 \lambda)^{2}}\right|
$$

Let $X=32(1+\lambda)(1+3 \lambda)(1+2 \lambda)^{2}$.

Using Lemma 2.2, it gives

$$
\left|a_{2} a_{4}-\mu a_{3}^{2}\right|=\frac{1}{X}\left|\begin{array}{l}
2\left[(1+2 \lambda)^{2}-(1+\lambda)(1+3 \lambda) \mu\right] p_{1}^{4} \\
+\left[3(1+2 \lambda)^{2}-4(1+\lambda)(1+3 \lambda) \mu\right]\left(4-p_{1}^{2}\right) p_{1}^{2} x \\
+2(1+2 \lambda)^{2} p_{1}\left(4-p_{1}^{2}\right)\left[1-|x|^{2}\right] z \\
-\left(4-p_{1}^{2}\right) x^{2}\left[\left[(1+2 \lambda)^{2}-2(1+\lambda)(1+3 \lambda) \mu\right] p_{1}^{2}\right. \\
+8(1+\lambda)(1+3 \lambda) \mu]
\end{array}\right|
$$

Suppose now that $p_{1}=p, p \in[0,2]$ and using triangle inequality, we get

$$
\left|a_{2} a_{4}-\mu a_{3}^{2}\right| \leq \frac{1}{X}\left[\begin{array}{l}
2\left|(1+2 \lambda)^{2}-(1+\lambda)(1+3 \lambda) \mu\right| p^{4} \\
+2(1+2 \lambda)^{2} p\left(4-p^{2}\right) \\
+\left|3(1+2 \lambda)^{2}-4(1+\lambda)(1+3 \lambda) \mu\right| p^{2}\left(4-p^{2}\right) \rho \\
+\left(4-p^{2}\right)\left(\left[\left|(1+2 \lambda)^{2}-2(1+\lambda)(1+3 \lambda) \mu\right| p^{2}\right.\right. \\
\left.+8|(1+\lambda)(1+3 \lambda) \mu|-2(1+2 \lambda)^{2} p\right) \rho^{2}
\end{array}\right]
$$

This gives rise to 


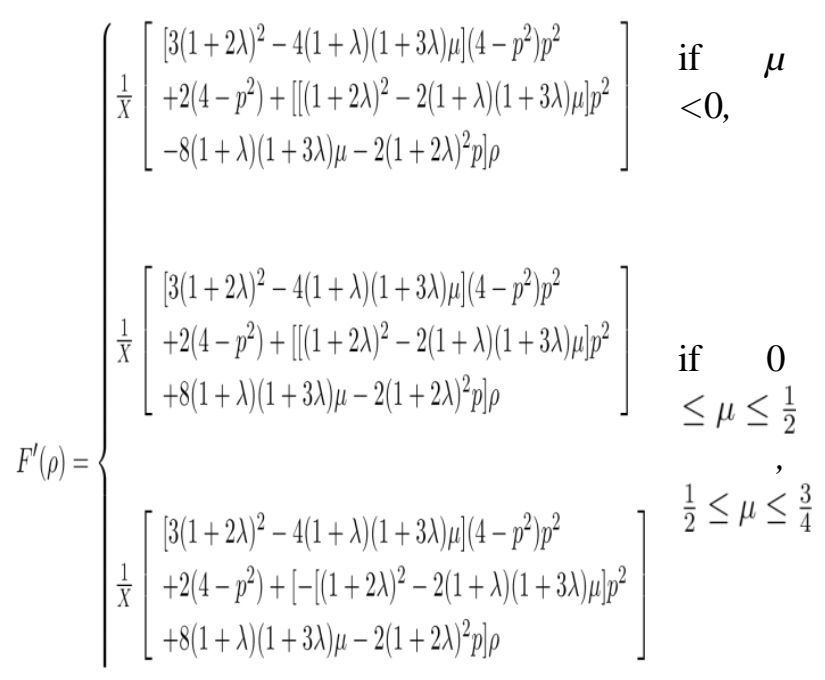

if,

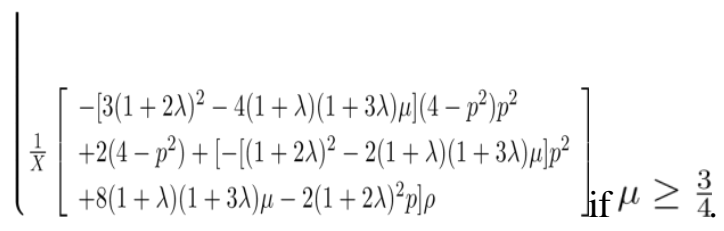

and for all the cases above, $F^{0}(\rho)>0$ for $\rho>0$; implying that

$$
\begin{aligned}
& \operatorname{Max} F(\rho)=F(1) \\
& \quad \text { Now let } \\
& G(p)=F(1)=\frac{1}{X}\left[\begin{array}{l}
2\left|(1+2 \lambda)^{2}-(1+\lambda)(1+3 \lambda) \mu\right| p^{4} \\
+2(1+2 \lambda)^{2} p\left(4-p^{2}\right)+\mid 3(1+2 \lambda)^{2} \\
-4(1+\lambda)(1+3 \lambda) \mu \mid p^{2}\left(4-p^{2}\right) \\
+\left(4-p^{2}\right)\left[(1+2 \lambda)^{2}-2(1+\lambda)(1+3 \lambda) \mu \mid p^{2}\right. \\
\left.+8|(1+\lambda)(1+3 \lambda) \mu|-2(1+2 \lambda)^{2} p\right]
\end{array}\right]
\end{aligned}
$$

Now we discuss the following cases:

Case I: For $\mu \leq 0$,

$G(p)=\frac{1}{X}\left[\begin{array}{l}-2\left[(1+2 \lambda)^{2}-2(1+\lambda)(1+3 \lambda) \mu\right] p^{4} \\ +16\left[(1+2 \lambda)^{2}-(1+\lambda)(1+3 \lambda) \mu\right] p^{2} \\ -32(1+\lambda)(1+3 \lambda) \mu\end{array}\right]$

and

$$
G^{\prime}(p)=\frac{1}{4(1+\lambda)(1+3 \lambda)(1+2 \lambda)^{2}} p\left[\begin{array}{l}
-\left[(1+2 \lambda)^{2}-2(1+\lambda)(1+3 \lambda) \mu\right] p^{2} \\
+4(1+2 \lambda)^{2}-4(1+\lambda)(1+3 \lambda) \mu
\end{array}\right]
$$

Easy calculation reveals that $G$ attains $p=2 \sqrt{\frac{(1+2 \lambda)^{2}-(1+\lambda)(1+3 \lambda) \mu}{(1+2 \lambda)^{2}-2(1+\lambda)(1+3 \lambda) \mu}}$.

The upper bound for equation (3.6) corresponds to $\rho=$ 1 and $p=2 \sqrt{\frac{(1+2 \lambda)^{2}-(1+\lambda)(1+3 \lambda) \mu}{(1+2 \lambda)^{2}-2(1+\lambda)(1+3 \lambda) \mu}}$, in which case

$$
\left|a_{2} a_{4}-\mu a_{3}^{2}\right| \leq \frac{\left[(1+2 \lambda)^{2}-(1+\lambda)(1+3 \lambda) \mu\right]^{2}}{\left[(1+2 \lambda)^{2}-2(1+\lambda)(1+3 \lambda) \mu\right]}-(1+\lambda)(1+3 \lambda) \mu
$$

Case II: For 0

$\leq \mu \leq \frac{1}{2}$

$G(p)=\frac{1}{16(1+\lambda)(1+3 \lambda)(1+2 \lambda)^{2}}\left[\begin{array}{l}{\left[-(1+2 \lambda)^{2}+2(1+\lambda)(1+3 \lambda) \mu\right] p^{4}} \\ +\left[8(1+2 \lambda)^{2}-16(1+\lambda)(1+3 \lambda) \mu\right] p^{2} \\ +16(1+\lambda)(1+3 \lambda) \mu\end{array}\right]$ and

$G^{\prime}(p)=\frac{1}{4(1+\lambda)(1+3 \lambda)(1+2 \lambda)^{2}} p\left[\begin{array}{l}-\left[(1+2 \lambda)^{2}-2(1+\lambda)(1+3 \lambda) \mu p^{2}\right. \\ +4(1+2 \lambda)^{2}-8(1+\lambda)(1+3 \lambda) \mu\end{array}\right]$

where $G$ attains its maximum vlaue at $p=2$, Hence we obtain

$$
\left|a_{2} a_{4}-\mu a_{3}^{2}\right| \leq(1+2 \lambda)^{2}-(1+\lambda)(1+3\rangle
$$

Case III: For $\frac{1}{2} \leq \mu \leq_{1 \text { we consider }}$ two subcases. Subcase (i): When

$$
\begin{aligned}
\frac{1}{2} \leq \mu & \leq \frac{3}{4} \\
G(p) & =\frac{1}{4(1+\lambda)(1+3 \lambda)(1+2 \lambda)^{2}}\left[\begin{array}{l}
{\left[(1+2 \lambda)^{2}-2(1+\lambda)(1+3 \lambda) \mu\right] p^{2}} \\
+4(1+\lambda)(1+3 \lambda) \mu
\end{array}\right]
\end{aligned}
$$

here $G$ attains its maximum value at $p=0$

Hence $\left|a_{2} a_{4}-\mu a_{3}^{2}\right| \leq(1+\lambda)(1+3 \lambda) \mu$

Subcase (ii): When

$$
\begin{aligned}
& \frac{3}{2} \leq \mu \leq 1 \\
& G(p)=\frac{1}{16(1+\lambda)(1+3 \lambda)(1+2 \lambda)^{2}}\left[\begin{array}{l}
{\left[3(1+2 \lambda)^{2}-4(1+\lambda)(1+3 \lambda) \mu\right] p^{4}} \\
+8\left[-(1+2 \lambda)^{2}+(1+\lambda)(1+3 \lambda) \mu\right] p^{2} \\
+16(1+\lambda)(1+3 \lambda) \mu
\end{array}\right]
\end{aligned}
$$

In this case $G(p)$ is a decreasing function so if attains its maximum value at $p=0$.

Case IV: Finally, for $\mu \geq 1$,

$$
G(p)=\frac{1}{16(1+\lambda)(1+3 \lambda)(1+2 \lambda)^{2}}\left[\begin{array}{l}
{\left[(1+2 \lambda)^{2}-2(1+\lambda)(1+3 \lambda) \mu\right] p^{4}} \\
+8\left[\mu(1+\lambda)(1+3 \lambda)-(1+2 \lambda)^{2}\right] p^{2} \\
+16(1+\lambda)(1+3 \lambda) \mu
\end{array}\right]
$$

and

$$
G^{\prime}(p)=\frac{1}{4(1+\lambda)(1+3 \lambda)(1+2 \lambda)^{2}} p\left[\begin{array}{l}
-\left[2(1+\lambda)(1+3 \lambda) \mu-(1+2 \lambda)^{2}\right] p^{2} \\
+4\left[(1+\lambda)(1+3 \lambda) \mu-(1+2 \lambda)^{2}\right]
\end{array}\right]
$$

Here $G$ attains its maximum

$$
\begin{aligned}
& \text { value at } p=2 \sqrt{\frac{\mu(1+\lambda)(1+3 \lambda)-(1+2 \lambda)^{2}}{2(1+\lambda)(1+3 \lambda) \mu-(1+2 \lambda)^{2}}} \text {. Hence } \\
& \qquad a_{2} a_{4}-\mu a_{3}^{2} \mid \leq \frac{\left[(1+\lambda)(1+3 \lambda) \mu-(1+2 \lambda)^{2}\right]^{2}}{\left[2(1+\lambda)(1+3 \lambda) \mu-(1+2 \lambda)^{2}\right]}+\mu(1+\lambda)(1+3 \lambda)
\end{aligned}
$$

For $\mu=1$, Theorem 3.1 gives the following result. its maximum value at Corollary 3.1.

If $f(z) \in C_{S(\lambda)}$ then $\left|a_{2} a_{4}-a_{3}^{2}\right| \leq(1+2 \lambda)^{2}$.

\section{Theorem 3.2.}

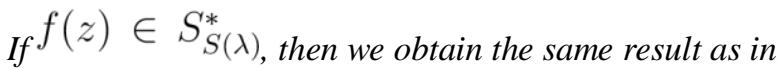
Theorem 3.1 on the same lines, we have

\section{Theorem 3.3.}

${ }_{\text {If }} f(z) \in K_{S(\lambda), \text { then }}^{*}$ 
$\left|a_{2} a_{4}-\mu a_{3}^{2}\right| \leq\left\{\begin{array}{lr}\frac{\left(9(1+2 \lambda)^{2}-8(1+\lambda)(1+3 \lambda) \mu\right)^{2}}{72\left(9(1+2 \lambda)^{2}-16(1+\lambda)(1+3 \lambda) \mu\right)}-\frac{1}{9} \mu(1+\lambda)(1+3 \lambda) & \text { if } \mu \leq 0, \\ \frac{1}{8}(1+2 \lambda)^{2}-\frac{1}{9}(1+\lambda)(1+3 \lambda) \mu & 0 \leq \mu \leq \frac{9}{16} \\ \frac{1}{9}(1+\lambda)(1+3 \lambda) \mu & \frac{9}{16} \leq \mu \leq \frac{9}{8},\end{array}\right.$

For $\mu=1$ Theorem 3.3 gives

\section{Corollary 3.2.}

If $f(z) \in K_{S(\lambda), \text { then }}\left|a_{2} a_{4}-a_{3}^{2}\right| \leq \frac{1}{9}$.

\section{Theorem 3.4.}

If $f(z) \in C_{S 1(\lambda)}$ then

$\int \frac{\left[135(1+2 \lambda)^{2}-98(1+\lambda)(1+3 \lambda) \mu\right]^{2}}{324\left[81(1+2 \lambda)^{2}-98(1+\lambda)(1+3 \lambda) \mu\right]}-\frac{41}{81}(1+\lambda)(1+3 \lambda) \mu \quad$ if $\mu \leq 0$

$\left|a_{2} a_{4}-\mu a_{3}^{2}\right| \leq \begin{cases}\frac{\left[135(1+2 \lambda)^{2}-196(1+\lambda)(1+3 \lambda) \mu\right)^{2}}{324\left(81(1+2 \lambda)^{2}-98(1+\lambda)(1+3 \lambda) \mu\right]}+\frac{49}{81}(1+\lambda)(1+3 \lambda) \mu & \text { if } \\ \frac{49}{81}(1+\lambda)(1+3 \lambda) \mu & 0 \leq \mu \leq \frac{135}{196}\end{cases}$

if $\frac{135}{196} \leq \mu \leq \frac{135}{98}$,

$\frac{\left[98(1+\lambda)(1+3 \lambda) \mu-135(1+2 \lambda)^{2}\right]^{2}}{324\left[98(1+\lambda)(1+3 \lambda) \mu-81(1+2 \lambda)^{2}\right]}+\frac{49}{81}(1+\lambda)(1+3 \lambda) \mu \quad$ if $\mu \geq \frac{135}{98}$.

Proof:

Since $f \in C_{S 1(\lambda)}$ by definition we have

$$
\frac{2\left[\lambda z^{2} f^{\prime \prime}(z)+z f^{\prime}(z)\right]}{\lambda z\left[h^{\prime}(z)+h^{\prime}(-z)\right]+(1-\lambda)[h(z)-h(-z)]}=p(z)
$$

where

$$
\infty h(z)=z+\mathrm{X} b k z k \in K S(\lambda)
$$

$k=2$

Using (1.1), (2.1) and (3.8), (3.9) gives

$$
\begin{aligned}
& 1+2(1+\lambda) a_{2} z+(1+2 \lambda) 3 a_{3} z^{2}+(1+3 \lambda) 4 a_{4} z^{3} \\
& =\left(1+p_{1} z+p_{2} z^{2}+p_{3} z^{3}+p_{4} z^{4}+\cdots\right)\left(1+(1+\lambda) b_{3} z^{2}+\cdots\right]
\end{aligned}
$$

On equating co-efficients in (3.10) we get

$$
\begin{gathered}
2(1+\lambda) a_{2}=p_{1}, \\
3 a_{3}(1+2 \lambda)=(1+2 \lambda) b_{3}+p_{2}, \\
4 a_{4}(1+3 \lambda)=(1+2 \lambda) p_{1} b_{3}+p_{3}
\end{gathered}
$$

From (3.9) we can easily verify that

$$
b_{3}=\frac{p_{2}}{6(1+2 \lambda)}
$$

So (3.11) yields

$$
a_{2}=\frac{p_{1}}{2(1+\lambda)}, \quad a_{3}=\frac{7 p_{2}}{18(1+2 \lambda)}, \quad a_{4}=\frac{p_{3}}{4(1+3 \lambda)}+\frac{p_{2} p_{1}}{24(1+3 \lambda)} \quad(3.12)
$$

From (3.12)

$$
\left|a_{2} a_{4}-\mu a_{3}^{2}\right|=\left|\frac{p_{1} p_{3}}{8(1+\lambda)(1+3 \lambda)}+\frac{p_{1}^{2} p_{2}}{48(1+\lambda)(1+3 \lambda)}-\mu \frac{49 p_{2}^{2}}{324(1+2 \lambda)^{2}}\right|
$$

Let $X_{1}=2592(1+\lambda)(1+3 \lambda)(1+2 \lambda)^{2}$.

Using Lemma 2.2 it gives

$$
\left|a_{2} a_{4}-\mu a_{3}^{2}\right| \leq \frac{1}{X_{1}}\left|\begin{array}{l}
{\left[108(1+2 \lambda)^{2}-98(1+\lambda)(1+3 \lambda) \mu\right] p_{1}^{4}} \\
+\left[189(1+2 \lambda)^{2}-196(1+\lambda)(1+3 \lambda) \mu\right]\left[4-p_{1}^{2}\right) p_{1}^{2} x \\
+162(1+2 \lambda)^{2}\left(4-p_{1}^{2}\right) p_{1}\left(1-|x|^{2}\right) z-\left(4-p_{1}^{2}\right) x^{2} \\
{\left[p_{1}^{2}\left[81(1+2 \lambda)^{2}-98(1+\lambda)(1+3 \lambda) \mu\right)\right.} \\
+392(1+\lambda)(1+3 \lambda) \mu
\end{array}\right|
$$

Suppose now that $p_{1}=p, p \in[0,2]$ and using triangle inequality we get

$$
\left|a_{2} a_{4}-\mu a_{3}^{2}\right| \leq \frac{1}{X_{1}}\left[\begin{array}{l}
\left.\mid 108(1+2 \lambda)^{2}-98(1+\lambda)(1+3 \lambda) \mu\right] p^{4} \\
+162(1+2 \lambda)^{2} p\left(4-p^{2}\right) \\
+\left|189(1+2 \lambda)^{2}-196(1+\lambda)(1+3 \lambda) \mu\right| p^{2}\left(4-p^{2}\right) \rho \\
+\left(4-p^{2}\right)\left(\left|81(1+2 \lambda)^{2}-98(1+\lambda)(1+3 \lambda) \mu\right| p^{2}\right. \\
\left.+392|(1+\lambda)(1+3 \lambda) \mu|-162(1+2 \lambda)^{2} p\right) \rho^{2}
\end{array}\right]
$$

This gives rise to 


$$
\begin{aligned}
G(p)=\frac{1}{1296(1+\lambda)(1+3 \lambda)(1+2 \lambda)^{2}} \\
\times\left[\begin{array}{l}
{\left[-81(1+2 \lambda)^{2}+98(1+\lambda)(1+3 \lambda) \mu\right] p^{4}} \\
+4\left[135(1+2 \lambda)^{2}-196(1+\lambda)(1+3 \lambda) \mu\right] p^{2} \\
+784(1+\lambda)(1+3 \lambda) \mu
\end{array}\right]
\end{aligned}
$$

We consider two subcases,

Subcase (i): When 0

$\leq \mu \leq \frac{135}{196}$

$G^{\prime}(p)=\frac{1}{324(1+\lambda)(1+3 \lambda)(1+2 \lambda)^{2}} p\left[\begin{array}{l}{\left[-81(1+2 \lambda)^{2}+98(1+\lambda)(1+3 \lambda) \mu p^{2}\right.} \\ \left.+270(1+2 \lambda)^{2}-392(1+\lambda)(1+3 \lambda) \mu\right]\end{array}\right]$

Here $G$ attains its maximum value at

$p=\sqrt{\frac{270(1+2 \lambda)^{2}-196(1+\lambda)(1+3 \lambda) \mu}{81(1+2 \lambda)^{2}-98(1+\lambda)(1+3 \lambda) \mu}}$

, in which case

$\left|a_{2} a_{4}-\mu a_{3}^{2}\right| \leq \frac{\left[135(1+2 \lambda)^{2}-196(1+\lambda)(1+3 \lambda) \mu\right]^{2}}{324\left[81(1+2 \lambda)^{2}-98(1+\lambda)(1+3 \lambda) \mu\right]}+\frac{49}{81}(1+\lambda)(1+3 \lambda) \mu$

Subcase (ii): When

$$
\begin{aligned}
\frac{135}{196} \leq \mu \leq & \frac{81}{98} \\
G(p)= & \frac{1}{1296(1+\lambda)(1+3 \lambda)(1+2 \lambda)^{2}} \\
\times & {\left[\begin{array}{l}
{\left[\begin{array}{l}
\left.-81(1+2 \lambda)^{2}+98(1+\lambda)(1+3 \lambda) \mu\right] p^{4} \\
-4\left[196(1+\lambda)(1+3 \lambda) \mu-135(1+2 \lambda)^{2}\right] p^{2} \\
+784(1+\lambda)(1+3 \lambda) \mu
\end{array}\right]}
\end{array}\right.}
\end{aligned}
$$

In this case $G(p)$ attains its maximum value at $p=0$. So

$$
\left|a_{2} a_{4}-\mu a_{3}^{2}\right| \leq \frac{49}{81}(1+\lambda)(1+3 \lambda) \mu
$$

Case III: For $\frac{81}{98} \leq \mu \leq \frac{108}{98}$, we consider two subcases.

\section{Subcase (i): When}

$$
\begin{aligned}
\frac{81}{98} \leq \mu & \leq \frac{189}{196} \\
G(p)= & \frac{1}{162(1+\lambda)(1+3 \lambda)(1+2 \lambda)^{2}} \\
& \times\left[\begin{array}{l}
{\left[27(1+2 \lambda)^{2}-49(1+\lambda)(1+3 \lambda) \mu\right] p^{2}} \\
+98(1+\lambda)(1+3 \lambda) \mu
\end{array}\right]
\end{aligned}
$$

here $G$ attains its maximum value at $p=0$. Hence

$$
\left|a_{2} a_{4}-\mu a_{3}^{2}\right| \leq \frac{49}{81}(1+\lambda)(1+3 \lambda) \mu
$$

Subcase (ii): When

$$
\begin{aligned}
& \frac{189}{196} \leq \mu \leq \frac{108}{98} \\
& G(p)=\frac{1}{1296(1+\lambda)(1+3 \lambda)(1+2 \lambda)^{2}} \\
& \times\left[\begin{array}{l}
{\left[189(1+2 \lambda)^{2}-196(1+\lambda)(1+3 \lambda) \mu\right] p^{4}} \\
+4\left[-135(1+2 \lambda)^{2}+98(1+\lambda)(1+3 \lambda) \mu\right] p^{2} \\
+784(1+\lambda)(1+3 \lambda) \mu
\end{array}\right]
\end{aligned}
$$

In this case $G(p)$ is a decreasing function so it attains its maximum value at $p=0$.

Case IV: Finally for $\mu \geq \frac{108}{98}$

$$
\begin{aligned}
G(p)=\frac{1}{1296(1+\lambda)(1+3 \lambda)(1+2 \lambda)^{2}} \\
\times\left[\begin{array}{l}
{\left[\left(81(1+2 \lambda)^{2}-98(1+\lambda)(1+3 \lambda) \mu\right] p^{4}\right.} \\
+4\left[98(1+\lambda)(1+3 \lambda) \mu-135(1+2 \lambda)^{2}\right] p^{2} \\
+784(1+\lambda)(1+3 \lambda) \mu
\end{array}\right]
\end{aligned}
$$

we consider two subcases:

Subcase (i): When $\frac{108}{98} \leq \mu \leq \frac{135}{98}, G(p)$ is a decreasing function so it attains its maximum value at $p=$ 0 .

Subcase (ii): When

$\mu \geq \frac{135}{98}$

$$
\begin{aligned}
G^{\prime}(p)= & \frac{1}{324(1+\lambda)(1+3 \lambda)(1+2 \lambda)^{2}} p \\
\times & {\left[\begin{array}{l}
{\left[81(1+2 \lambda)^{2}-98(1+\lambda)(1+3 \lambda) \mu\right] p^{2}} \\
+2\left[98(1+\lambda)(1+3 \lambda) \mu-135(1+2 \lambda)^{2}\right.
\end{array}\right] }
\end{aligned}
$$

here $G$ attains its maximum value

${ }_{\text {at }} p=\sqrt{\frac{196(1+\lambda)(1+3 \lambda) \mu-270(1+2 \lambda)^{2}}{98(1+\lambda)(1+3 \lambda) \mu-81(1+2 \lambda)^{2}}}$.

Hence

$\left|a_{2} a_{4}-\mu a_{3}^{2}\right| \leq \frac{\left[98(1+\lambda)(1+3 \lambda) \mu-135(1+2 \lambda)^{2}\right]^{2}}{324\left[98(1+\lambda)(1+3 \lambda) \mu-81(1+2 \lambda)^{2}\right]}+\frac{49}{81}(1+\lambda)(1+3 \lambda) \mu$

For $\mu=1$, Theorem 3.4 gives the following result.

\section{Corollary 3.3.}

If $f(z) \in C_{S 1(\lambda)}$ then $\left|a_{2} a_{4}-a_{3}^{2}\right| \leq \frac{49}{81}(1+2 \lambda)^{2}$ on the same lines, we can easily prove the following theorem:

\section{Theorem 3.5.}

If $f(z) \in C_{S 1(\lambda)}^{\prime}$ then we get the same result as in Theorem 3.3 .

\section{Theorem 3.6.}

If $f(z) \in C_{S(\gamma, \lambda)}$ then

$$
\left|a_{2} a_{4}-\mu a_{3}^{2}\right| \leq \begin{cases}\frac{\left[A_{1}^{2}-B_{1} C_{1} \mu\right]^{2}}{\left[A_{1}^{2}-2 B_{1} C_{1} \mu\right]}-B_{1} C_{1} \mu & i \mu \leq 0, \\ A_{1}^{2}-B_{1} C_{1} \mu & \text { if } \\ B_{1} C_{1} \mu & 0 \leq \mu \leq \frac{1}{2}\end{cases}
$$$$
\frac{1}{2} \leq \mu \leq 1,
$$

$\mid \frac{\left[B_{1} C_{1} \mu-A_{1}^{2}\right]^{2}}{\left[2 B_{1} C_{1} \mu-A_{1}^{2}\right]}+B_{1} C_{1} \mu$

if $\mu \geq 1$.

where $A_{1}=(1+6 \gamma \lambda+2 \gamma-2 \lambda)$

$$
\begin{aligned}
& B_{1}=(1+12 \gamma \lambda+3 \gamma-3 \lambda) \\
& C_{1}=(1+2 \gamma \lambda+\gamma-\lambda)
\end{aligned}
$$

Proof:

Since $f \in C_{S(\gamma, \lambda)}$ we have

$$
\frac{2\left[\gamma \lambda z^{3} f^{\prime \prime \prime}(z)+(2 \gamma \lambda+\gamma-\lambda) z^{2} f^{\prime \prime}(z)+z f^{\prime}(z)\right.}{\gamma \lambda z^{2}\left[g^{\prime \prime}(z)-g^{\prime \prime}(-z)\right]+(\gamma-\lambda) z\left[g^{\prime}(z)+g^{\prime}(-z)\right]+(1-\gamma+\lambda)[g(z)-g(-z)]}=p(z)
$$

where 


$$
g(z)=z+\underset{k=2}{X} b k z k \in S S *(\gamma, \lambda)
$$

Using (1.1), (2.1) and (3.15), (3.16) gives

$$
\begin{aligned}
1+2( & 2 \gamma \lambda+\gamma-\lambda+1) a_{2} z+3 a_{3}[6 \gamma \lambda+2 \gamma-2 \lambda+1] z^{2} \\
& +4 a_{4}[1+6 \gamma \lambda+3(2 \gamma \lambda+\gamma-\lambda)] z^{3}+\cdots \\
& =\left(1+p_{1} z+p_{2} z^{2}+\cdots\right)\left(1+b_{3}(1+6 \gamma \lambda+2 \gamma-2 \lambda) z^{2}+\cdots\right)
\end{aligned}
$$

On equating coefficients in (3.17) we get

$$
\begin{gathered}
2[2 \gamma \lambda+\gamma-\lambda+1) a_{2}=p_{1}, \\
3 a_{3}[6 \gamma \lambda+2 \gamma-2 \lambda+1]=b_{3}[1+6 \gamma \lambda+2 \gamma-2 \lambda)+p_{2}, \\
4 a_{4}[1+6 \gamma \lambda+3(2 \gamma \lambda+\gamma-\lambda)]=p_{1} b_{3}[1+6 \gamma \lambda+2 \gamma-2 \lambda)+p_{3}
\end{gathered}
$$

From (3.16), we can easily verify that

$b_{3}=\frac{p_{2}}{2[1+2 \gamma-2 \lambda+6 \gamma \lambda]}$

So (3.18) yields

$$
\begin{aligned}
a_{2} & =\frac{p_{1}}{2[1+2 \gamma \lambda+\gamma-\lambda]}, \\
a_{3} & =\frac{p_{2}}{2[1+6 \gamma \lambda+2 \gamma-2 \lambda]}, \\
a_{4} & =\frac{p_{1} p_{2}}{8[1+12 \gamma \lambda+3 \gamma-3 \lambda]}+\frac{p_{3}}{4[1+12 \gamma \lambda+3 \gamma-3 \lambda]}
\end{aligned}
$$$$
\frac{1}{32 A_{1}^{2} B_{1} C_{1}}\left[\begin{array}{c}
-\left[3 A_{1}^{2}-4 B_{1} C_{1} \mu\right]\left(4-p^{2}\right) p^{2}+2\left(4-p^{2}\right) \\
{\left[-\left(A_{1}^{2}-2 B_{1} C_{1} \mu\right) p^{2}+8 B_{1} C_{1} \mu-2 A_{1}^{2} p\right] \rho}
\end{array}\right]
$$

From (3.20) define

$A_{1}=(1+6 \gamma \lambda+2 \gamma-2 \lambda)$

$B_{1}=(1+12 \gamma \lambda+3 \gamma-3 \lambda)$

$C_{1}=(1+2 \gamma \lambda+\gamma-\lambda)$

$$
\left|a_{2} a_{4}-\mu a_{3}^{2}\right| \leq\left|\frac{p_{1}^{2} p_{2}}{16 B_{1} C_{1}}+\frac{p_{1} p_{3}}{8 B_{1} C_{1}}-\mu \frac{p_{2}^{2}}{4 A_{1}^{2}}\right|
$$

Using Lemma 2.2 it gives

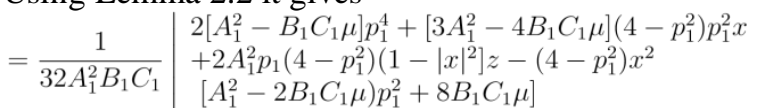

Suppose now that $p_{1}=p, p \in[0,2]$ and using triangle inequality, we get

$$
=F(\rho) \text { with } \rho=|x| \leq 1
$$

This gives rise to

$$
\begin{gathered}
\left|a_{2} a_{4}-\mu a_{3}^{2}\right| \leq \frac{1}{32 A_{1}^{2} B_{1} C_{1}}\left[\begin{array}{l}
2\left|A_{1}^{2}-B_{1} C_{1} \mu\right| p_{1}^{4}+2 A_{1}^{2} p\left(4-p_{1}^{2}\right) \\
+\left|3 A_{1}^{2}-4 B_{1} C_{1} \mu\right| p^{2}\left(4-p^{2}\right) \rho+\left(4-p^{2}\right) \\
{\left[\left|A_{1}^{2}-2 B_{1} C_{1} \mu\right| p^{2}+8\left|B_{1} C_{1} \mu\right|-2 A_{1}^{2} p\right] \rho^{2}}
\end{array}\right] \\
\text { if } \boldsymbol{\mu} \leq \mu \leq \frac{1}{2}<0
\end{gathered}
$$

if 0 ,

if $\frac{1}{2} \leq \mu \leq \frac{3}{4}$
$F^{\prime}(\rho)=\left\{\begin{array}{c}\frac{1}{32 A_{1}^{2} B_{1} C_{1}}\left[\begin{array}{l}{\left[3 A_{1}^{2}-4 B_{1} C_{1} \mu\right]\left(4-p^{2}\right) p^{2}+2\left(4-p^{2}\right)} \\ {\left[\left(A_{1}^{2}-2 B_{1} C_{1} \mu\right) p^{2}-8 B_{1} C_{1} \mu-2 A_{1}^{2} p\right] \rho}\end{array}\right] \\ \frac{1}{32 A_{1}^{2} B_{1} C_{1}}\left[\begin{array}{l}{\left[3 A_{1}^{2}-4 B_{1} C_{1} \mu\right]\left(4-p^{2}\right) p^{2}+2\left(4-p^{2}\right)} \\ {\left[\left(A_{1}^{2}-2 B_{1} C_{1} \mu\right) p^{2}+8 B_{1} C_{1} \mu-2 A_{1}^{2} p\right] \rho}\end{array}\right] \\ \frac{1}{32 A_{1}^{2} B_{1} C_{1}}\left[\begin{array}{l}{\left[3 A_{1}^{2}-4 B_{1} C_{1} \mu\right]\left(4-p^{2}\right) p^{2}+2\left(4-p^{2}\right)} \\ {\left[-\left(A_{1}^{2}-2 B_{1} C_{1} \mu\right) p^{2}+8 B_{1} C_{1} \mu-2 A_{1}^{2} p\right] \rho}\end{array}\right]\end{array}\right.$

$\mu \geq \frac{3}{4}$

and for all the cases above $F^{0}(\rho)>0$ for $\rho>0$, implying that Max $F(\rho)=F(1)$ Now let $\quad(3.20)$

$$
G(P)=F(1)=\frac{1}{32 A_{1}^{2} B_{1} C_{1}}\left[\begin{array}{l}
2\left|A_{1}^{2}-B_{1} C_{1} \mu\right| p^{4}+2 A_{1}^{2} p\left(4-p^{2}\right) \\
+\left|3 A_{1}^{2}-4 B_{1} C_{1} \mu\right| p^{2}\left(4-p^{2}\right) \\
+\left(4-p^{2}\right)\left[\left|A_{1}^{2}-2 B_{1} C_{1} \mu\right| p^{2}+8\left|B_{1} C_{1} \mu\right|-2 A_{1}^{2} p\right]
\end{array}\right]
$$

Now we discuss the following cases:

Case I: For $\mu \leq 0$,

$G(P)=\frac{1}{32 A_{1}^{2} B_{1} C_{1}}\left[-2\left[A_{1}^{2}-2 B_{1} C_{1} \mu\right] p^{4}+16\left[A_{1}^{2}-B_{1} C_{1} \mu\right] p^{2}-32 B_{1} C_{1} \mu\right]$

and

$$
G^{\prime}(P)=\frac{1}{4 A_{1}^{2} B_{1} C_{1}} p\left[-\left[A_{1}^{2}-2 B_{1} C_{1} \mu\right] p^{2}+4 A_{1}^{2}-4 B_{1} C_{1} \mu\right]
$$

Easy calculation reveals that $G$ attainsits

$$
p=2 \sqrt{\frac{A_{1}^{2}-B_{1} C_{1} \mu}{A_{1}^{2}-2 B_{1} C_{1} \mu}} \text { value at }
$$

The upper bound for equation (3.21) corresponds to $\rho$ $=1$ and $p=2 \sqrt{\frac{A_{1}^{2}-B_{1} C_{1} \mu}{A_{1}^{2}-2 B_{1} C_{1} \mu}}$, in which case $\left|\log _{2} Q_{4}-\mu 0_{3}^{2}\right| \leq \frac{\left|A_{1}^{2}-B_{1} C_{1}\right|^{2}}{A_{1}^{2}-2 B_{1} C_{1} \mu}-B_{1} C_{1} \mu$

Case II: For $0 \leq \mu \leq \frac{1}{2}$, 
$G(P)=\frac{1}{16 A_{1}^{2} B_{1} C_{1}}\left[\left(-A_{1}^{2}+2 B_{1} C_{1} \mu\right) p^{4}+\left[8 A_{1}^{2}-16 B_{1} C_{1} \mu\right] p^{2}+16 B_{1} C_{1} \mu\right.$
$G^{\prime}(P)=\frac{1}{4 A_{1}^{2} B_{1} C_{1}} p\left[-\left(A_{1}^{2}-2 B_{1} C_{1}\right) p^{2}+4 A_{1}^{2}-8 B_{1} C_{1} \mu\right]$

where $G$ attains maximum value at $p=2$,

Hence $\left|a_{2} a_{4}-\mu a_{3}^{2}\right| \leq A_{1}^{2}-B_{1} C_{1} \mu$.

Case III: For $\quad \frac{1}{2} \leq \mu \leq_{1}$, we consider two subcases.

Subcase (i):

$\frac{1}{2} \leq \mu \leq \frac{3}{4}$
$\left.G(P)=\frac{1}{4 A_{1}^{2} B_{1} C_{1}}\left[\left(A_{1}^{2}-2 B_{1} C_{1} \mu\right) p^{2}+4 B_{1} C_{1} \mu\right]\right]$

Hence $G$ attains its maximum value at $p=0$ hence

$\left|a_{2} a_{4}-\mu a_{3}^{2}\right| \leq B_{1} C_{1} \mu$.

Subcase (ii): $\quad \frac{3}{4} \leq \mu \leq 1$,

$G(P)=\frac{1}{16 A_{1}^{2} B_{1} C_{1}}\left[\left(3 A_{1}^{2}-4 B_{1} C_{1} \mu\right) p^{4}+8\left(-A_{1}^{2}+B_{1} C_{1} \mu\right) p^{2}+\right.$

In this case $G(p)$ is a decreasing function so it attains its maximum value at $p=0$.

Case IV: Finally, for $\mu \geq 1$,

$$
G(P)=\frac{1}{16 A_{1}^{2} B_{1} C_{1}}\left[\left(A_{1}^{2}-2 B_{1} C_{1} \mu\right) p^{4}+8\left(B_{1} C_{1} \mu-A_{1}^{2}\right) p^{2}+16 B_{1} C_{1} \mu\right]
$$

and

$G^{\prime}(P)=\frac{1}{4 A_{1}^{2} B_{1} C_{1}} p\left[-\left(2 B_{1} C_{1} \mu-A_{1}^{2}\right) p^{2}+4\left(B_{1} C_{1} \mu-A_{1}^{2}\right)\right]$

here $G$ attains maximum value at $p=2 \sqrt{\frac{B_{1} C_{1} \mu-A_{1}^{2}}{2 B_{1} C_{1} \mu-A_{1}^{2}}}$.

Hence $\left|a_{2} a_{4}-\mu a_{3}^{2}\right| \leq \frac{\left(B_{1} C_{1} \mu-A_{1}^{2}\right)^{2}}{\left(2 B_{1} C_{1} \mu-A^{2}\right)}+\mu B_{1} C_{1}$.

For $\mu$

$=1$, Theorem 3.6

gives the following result:

\section{Corollary 3.4.}

If $f(z) \in C_{S(\gamma, \lambda)}$, then $\left|a_{2} a_{4}-a_{3}^{2}\right| \leq A_{1}^{2}$.

\section{Theorem 3.7.}

If $f(z) \in S_{S^{*}(\gamma, \lambda)}$ then we obtain the same result as in Theorem 3.6. On the same lines we have

\section{Theorem 3.8.}

If $f(z) \in K_{S(\gamma, \lambda)}$ then

$$
\begin{gathered}
\int \frac{\left(9 A_{1}^{2}-8 B_{1} C_{1} \mu\right)^{2}}{72\left(9 A_{1}^{2}-16 B_{1} C_{1} \mu\right)}-\frac{1}{9} B_{1} C_{1} \mu \\
\text { if } \mu \leq 0, \\
\text { if } 0 \leq \mu \leq \frac{9}{16}, \text { if } \frac{9}{16} \leq \mu \leq \frac{9}{8}, \\
\text { if } \mu \geq \frac{9}{8} .
\end{gathered}
$$

For $\mu=1$ Theorem 3.8 gives:

\section{Corollary 3.5 .}

If $f(z) \in K_{S(\gamma, \lambda)}$, then $\left|a_{2} a_{4}-a_{3}^{2}\right| \leq \frac{A_{1}^{2}}{9}$.

Theorem 3.9. If $f(z) \in C_{S 2(\gamma, \lambda)}$, then

$$
\left|a_{2} a_{4}-\mu a_{3}^{2}\right| \leq\left\{\begin{array}{l}
\frac{\left(135 A_{1}^{2}-19 \mu B_{1} C_{1}\right)^{2}}{324\left(81 A_{1}^{2}-98 B_{1} C_{1} \mu\right)}+\frac{49}{81} B_{1} C_{1} \mu \\
\frac{49}{81} B_{1} C_{1} \mu
\end{array}\right.
$$

$$
\begin{aligned}
& \left(\frac{\left(135 A_{1}^{2}-98 B_{1} C_{1} \mu\right)^{2}}{324\left(81 A_{1}^{2}-98 B_{1} C_{1} \mu\right)}-\frac{49}{81} B_{1} C_{1} \mu\right. \\
& \text { if } \mu \leq 0, \\
& \left(\frac{\left(98 B_{1} C_{1} \mu-135 A_{1}^{2}\right)^{2}}{324\left(98 B_{1} C_{1} \mu-81 A_{1}^{2}\right)}+\frac{49}{81} B_{1} C_{1} \mu \quad \text { if } \mu \geq \frac{135}{98} .\right.
\end{aligned}
$$

For $\mu=1$ Theorem 3.9 gives the following result:

\section{Corollary 3.6.}

If $f(z) \in C_{S 2(\gamma, \lambda) \text {, then }}\left|a_{2} a_{4}-a_{3}^{2}\right| \leq \frac{49}{81} A_{1}^{2}$ on the same lines we can easily prove the following theorem:

Theorem 3.10.

If $f(z) \in C_{S 2(\gamma, \lambda) \text {, then we get the same result as in }}^{\prime}$

Theorem 3.8.

\section{REFERENCES}

1. Chuah Puoi Choo and Aini Janteng, Estimate on the Second Hankel functional for a subclass of close-to-convex functions with respect to symmetric points, Int. J. of Math. Anal., 7(16) (2013), 781-788.

2. R.N. Das and P. Singh, On subclasses of Schlicht mappings, Indian J. Pure Appl. Math., 8 (1977), 864-872.

3. R. Ehrenborg, The Hankel determinant of exponential polynomials, American Mathematical Monthly, 107 (2000), 557-560.

4. M. Fekete and G. Szeg“o, Eine Bemerkung Uber ungerade Schlichte Funktionen, J. London Math. Soc., 8 (1933), 85-89.

5. W.K. Hayman, Multivalent functions, Cambridge Tracts in Math. and Math. Phys., 48, Cambridge University Press, Cambridge, 1958.

6. J.W. Layman, The Hankel transform and some of its properties, J. of Integer Sequences, 4 (2001), 1-11.

7. R.J. Libera and E.-J. Zlotkiewiez, Early coefficients of the inverse of a regular convex function, Proc. Amer. Math. Soc., 85 (1982), 225-230.

8. R.J. Libera and E.-J. Zlotkiewiez, Coefficient bounds for the inverse of a function with derivative in P, Proc. Amer. Math. Soc., 87 (1983), 251-257.

9. B.S. Mehrok and Gagandeep Singh, Estimate of second Hankel determinant for certain classes of analytic functions, Scientia Magna, 8(3) (2012), 85-94.

10. Aini Janteng, Suzeini Abdul Halim and Maslina Darus, Hankel determinant for starlike and convex functions, Int. J. Math. Anal., 1(13) (2007), 619-625.

11. Aini Janteng, Suzeini Abdul Halim and Maslina Darus, Hankel determinant for functions starlike and convex with respect to symmetric points, J. Quality Measurement and Anal., 2(1) (2006), 37-43.

12. J.W. Noonan and D.K. Thomas, On the second Hankel determinant of a really mean p-valent functions, Trans. Amer. Math. Soc., 223(2) (1976), 337-346.

13. K.I. Noor, Hankel determinant problem for the class of functions with bounded boundary rotation, Rev. Roum. Math. Pures Et Appl., 28(8) (1983), 731-739.

14. Ch. Pommerenke, Univalent functions, G“ottingen, Vandenhoeck and Ruprecht, 1975

15. M.O. Reade, On close-to-convex univalent functions, Michigan Math. J., 3 (1955), 59-62.

16. K. Sakaguchi, On a certain univalent mapping, J. Math. Soc. Japan, 11 (1959), 72-80

\section{AUTHORS PROFILE}

B. Srutha Keerthi ${ }^{1}$ School of Advanced Sciences Mathematics Division, VIT University, Chennai Campus

Vandalur, Kelambakkam, Chennai - 600127 sruthilaya06@yahoo.co.in

S.Prema, ${ }^{2}$ Department of Mathematics SRM Institute of Science and Technology $\begin{array}{lll}\text { Chennai } \quad-\quad 600 & 089\end{array}$ cs.prem@yahoo.co.in 